\title{
Maduración y comportamiento poscosecha de la guayaba (Psidium guajava L.). Una revisión
}

\section{Ripening and postharvest behavior in guava (Psidium guajava L.). A review}

ALFONSO PARRA-CORONADO1, 2

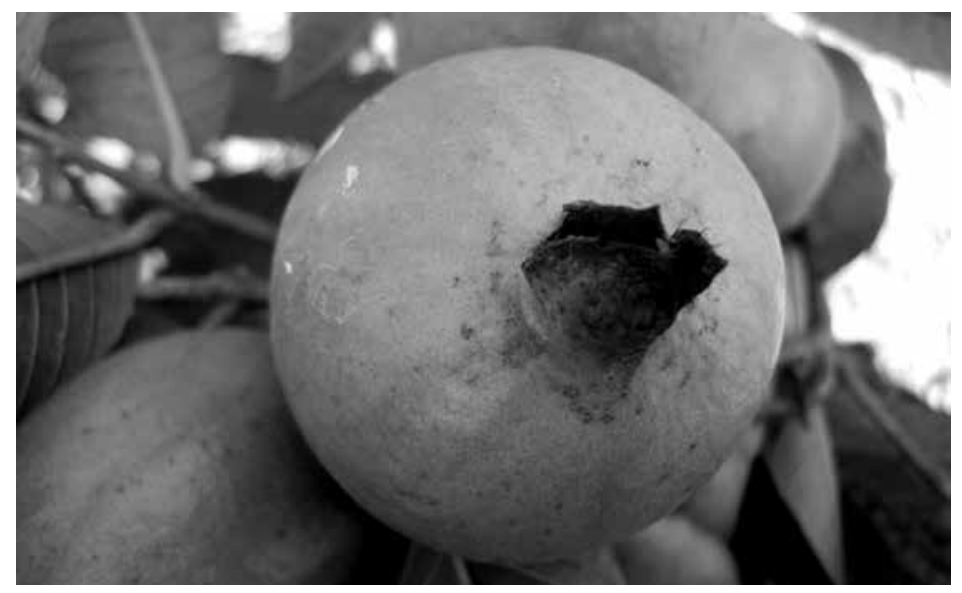

Guayaba manzana en maduración.

Foto: G. Fischer

\section{RESUMEN}

Dentro de la familia Myrtaceae, la guayaba es la principal especie del género Psidium por su importancia económica. La clasificación de la guayaba como fruto climatérico o no climatérico es contradictoria, depende de la variedad. Los diferentes estudios indican que a medida que transcurre el periodo poscosecha, se presenta pérdida de peso, aumento en los sólidos solubles y el pH, además se disminuye la firmeza del fruto y el ángulo hue, el cual expresa significativamente el cambio en el color de la epidermis y es un buen indicador del estado de madurez. La tendencia de la acidez titulable y del contenido de ácido ascórbico son muy variables, aumentando y/o disminuyendo durante la poscosecha, según características varietales. Los frutos de la guayaba son muy susceptibles al daño por frío y a enfermedades generándose pardeamiento (temperatura óptima de almacenamiento $5-10^{\circ} \mathrm{C}$ ). El comportamiento poscosecha está influenciado por las características varietales, la edad de la planta y por las condiciones climáticas y de cultivo a que hayan estado expuestos.

Palabras clave adicionales: climatérico, índice de madurez, cambios físicos, cambios bioquímicos, Myrtaceae.

\footnotetext{
Facultad de Ingeniería, Departamento de Ingeniería Civil y Agrícola, Universidad Nacional de Colombia, Bogotá (Colombia).

2 Autor para correspondencia..aparrac@unal.edu.co
} 


\section{ABSTRACT}

Within the Myrtaceae family, guava is the main species of the Psidium genus because of its economic importance. The classification of guava as a climacteric or non-climacteric fruit is contradictory, varying according to the variety. Different postharvest studies have indicated that, as the postharvest period elapses, weight loss, soluble solids and $\mathrm{pH}$ increase and fruit firmness and hue angle decrease. Hue angle significantly expresses the change in the color of the skin and is a good indicator of maturity. The trends of titratable acidity and ascorbic acid content are highly variable, increasing and/or decreasing in the postharvest according to the varietal characteristics. The guava fruits are very susceptible to chilling injury and disease (optimal storage temperature is $5-10^{\circ} \mathrm{C}$ ). The postharvest behavior is influenced by varietal characteristics, the age of the plant and the climatic and growing conditions.

Additional key words: climacteric, maturity index, physical changes, biochemical changes, Myrtaceae.

Fecha de recepción: 04-06-2014

Aprobado para publicación: 10-11-2014
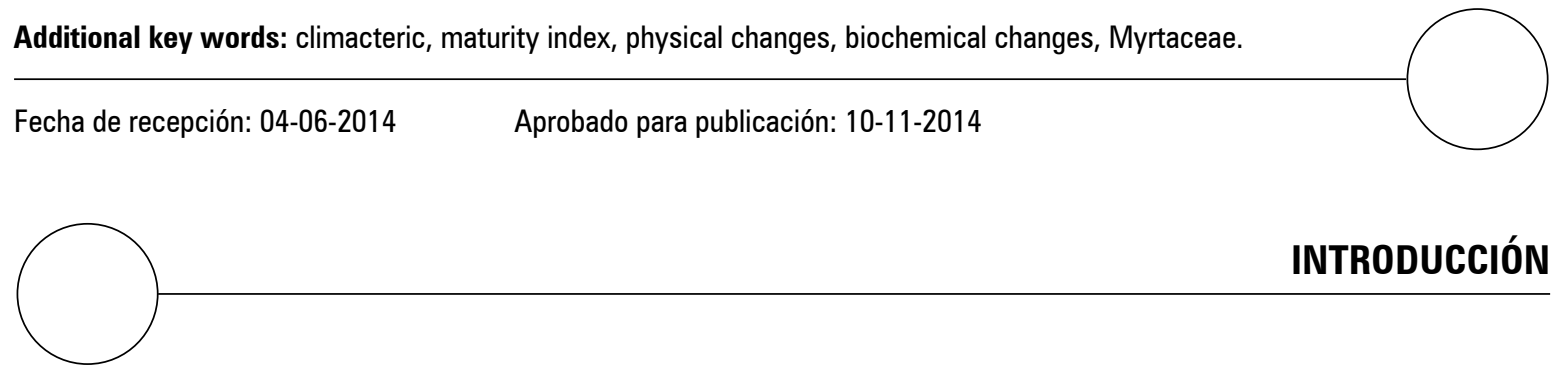

INTRODUCCIÓN

La familia Myrtaceae está representada por aproximadamente 133 géneros y 3.800 especies. En el género Psidium, representado por aproximadamente 150 especies, se destaca la guayaba por su importancia económica (Wilson et al., 2001). La guayaba (Psidium guajava L.), se encuentra entre los 0 a $2.000 \mathrm{msnm}$, en una amplia variedad de climas, con precipitaciones de 1.000 a $2.000 \mathrm{~mm}_{\text {nño- }}{ }^{-1}$, temperaturas medias entre 20 y $30^{\circ} \mathrm{C}$; requiere buena exposición solar (Solarte et al., 2010a). Se cultiva comercialmente en muchos países tropicales y subtropicales del mundo, siendo India el mayor productor, seguido por Pakistán, México y Brasil, además son países productores Egipto, Tailandia, Colombia, Indonesia, Venezuela Sudan, Bangladesh, Cuba, Vietnam, Malasia, Puerto Rico, Australia y Estados Unidos (Singh, 2011).

Según Yam Tzec et al. (2010), la producción mundial de guayaba es aproximadamente de 1,2 millones de toneladas, la India y Pakistán aportan el $50 \%$, México el $25 \%$ y el resto lo aportan otros países como Colombia, Egipto y Brasil. Durante el año 2013 se cosecharon en Colombia 8.339 ha de guayaba, con producción total de 69.096 t, distribuida en 12 departamentos, siendo los principales departamentos productores: Santander, Tolima, Boyacá, Valle del Cauca, Antioquia y Caldas. El área del cultivo está bajando considerando que en el año 2000 hubo 12.603 ha, en 200512.920 ha y en 20109.810 ha en producción (Agronet, 2014).

Los cultivares Paluma, Rica, Pedro Sato, Ogawa, IAC-3, IAC-4, Sassaoka, Kumagai y Século XXI son de pulpa roja, mientras que los cultivares Iwao, Kumagai y White Selection of Florida son de pulpa blanca (Pereira et al., 2003; Pereira y Nachtigal, 2009). En Brasil, los cultivares rojos de piel rugosa, principalmente Pedro Sato, Sassoka y Paluma, son los más plantados para la producción de fruta fresca; estos cultivares y la variedad Kumagai de pulpa blanca, son la base de la exportación brasileña de guayaba (Mendes-Pereira y Kavati, 2011). En Colombia se cultivan diferentes variedades, tales como: Regional Blanca, Regional Roja, Ráquira Blanca, Guavatá Victoria, Pera y Manzana (Solarte et al., 2010b).

El uso popular de la guayaba en productos elaborados tales como néctar, jugo, conservas, 
mermeladas, fruta en almíbar, alimentos para niños, refrescos, lácteos y panadería, la convierten en una de las frutas favoritas de miles de millones de personas en todo el mundo, particularmente en los trópicos y en los subtrópicos cálidos y se están volviendo cada vez más populares en los mercados de Europa y América del Norte (Pérez-Gutiérrez et al., 2008; Vieira et al., 2008; Singh, 2011).

Desde el punto de vista nutricional, los frutos de guayaba son una excelente fuente de antioxidantes, tales como ácido ascórbico (Lim et al., 2007; Restrepo-Sánchez et al., 2009; Batista et al., 2012a; Fischer et al., 2012), carotenoides y fenoles (Lim et al., 2007), los cuales cumplen un papel importante en la prevención de enfermedades crónicas y degenerativas (Espinal et al., 2010). También es abundante en fibra dietética (48-49\% en base a materia seca), vitamina A, vitaminas del grupo B (tiamina y niacina) pectina, fósforo, calcio, hierro y potasio; tiene alto contenido de licopeno y posee características funcionales anticancerígenas (Vieira et al., 2008; Singh, 2011; Batista et al., 2012a; Fischer et al., 2012). Los compuestos orgánicos volátiles predominantes incluyen los ácidos cítrico, láctico, málico, ascórbico y galacturónico, cuyo contenido cambia dependiendo de los cultivares y las condiciones de cultivo (Batista et al., 2012a).

Durante la poscosecha se producen en los frutos una serie de cambios, generalmente coordinados, tanto de síntesis como de degradación, que son controlados genéticamente y que conducen a la senescencia (Kader, 2002). Los cambios, aunque son muy variables entre las especies, incluyen generalmente la modificación de la ultraestructura y textura de la pared celular, así como cambios en: turgencia, jugosidad, conversión de almidones en azúcares, incremento en la susceptibilidad a patógenos en la poscosecha, alteraciones en la biosíntesis de pigmentos y de compuestos responsables del sabor (Giovannoni, 2001); estos cambios son los que van a determinar la calidad poscosecha de los frutos.
Durante el proceso de maduración las guayabas presentan cambios en algunas variables como la firmeza, el peso y el color, logrando obtener tablas de calidad de las diferentes variedades de guayaba en función de la escala de color (Solarte et al., 2010b).

Debido al volumen de producción y la importancia socioeconómica del cultivo en muchos departamentos de Colombia $y$, teniendo en cuenta que es un fruto altamente perecedero, es muy significativo conocer el comportamiento poscosecha con el fin de realizar un manejo adecuado de los frutos, encaminado a conservar su calidad, que es el objetivo de esta revisión.

\section{MADURACIÓN EN PLANTA E ÍNDICES DE MADUREZ}

La identificación del grado de madurez apropiado para realizar la cosecha del fruto es de gran importancia ya que repercute en la vida poscosecha del mismo, así como en su comercialización (Parra-Coronado y Hernández-Hernández, 2008). En regiones tropicales, la guayaba presenta producción durante todo el año, presentándose la cosecha entre 120 a 180 d después de la floración, dependiendo de la variedad, de la época del año y de las condiciones agroecológicas y de cultivo (Solarte et al., 2010c; Yadava, 1996). Mercado-Silva et al. (1998) encontraron que el fruto de guayaba variedad Media China en la temporada primavera-verano requirió 130 d desde floración hasta la cosecha, mientras que el fruto en la temporada de otoño-invierno necesito 190 d para alcanzar la madurez.

No hay consenso respecto al estado ideal de madurez de este fruto y su cosecha se realiza teniendo en cuenta aspectos comerciales, más que fisiológicos, cuando el fruto está firme e inicia el cambio de color verde oscuro a amarillento (Kader, 2002; Fischer et al., 2012. Solarte et al. (2010b) encontraron que el color es un buen índice de cosecha y que el punto de esta 
se basa en evaluaciones subjetivas de tamaño del fruto, color de la epidermis y firmeza; sin embargo, estas características pueden variar en una misma localidad de acuerdo con el cultivar, la edad de la planta, la época del año, los procedimientos de manejo y la interacción entre estos factores $\mathrm{u}$ otros.

\section{MADURACIÓN POSCOSECHA DEL FRUTO}

\section{Cambios fisiológicos}

Los cambios fisiológicos que se presentan en el proceso de maduración poscosecha de los frutos están influenciados por el estado de madurez en la cosecha y por las condiciones climáticas predominantes en el cultivo y por las condiciones de manejo y almacenamiento poscosecha (Wills et al., 2007). En muchos frutos, el inicio de la maduración después de la recolección oportuna, está acompañado por un aumento en la intensidad respiratoria y en la producción de etileno, alcanzando su máximo valor cuando el fruto está completamente maduro y disminuyendo durante la senescencia; los frutos que presentan este comportamiento se les denomina climatéricos; los frutos no climatéricos no presentan aumento de la respiración (Kays y Paull, 2004). El comportamiento fisiológico poscosecha de los productos hortofrutícolas está influenciado por las condiciones de almacenamiento de los mismos, siendo la temperatura de almacenamiento el principal factor (Parra-Coronado y Hernández-Hernández, 2008).

\section{Intensidad respiratoria (IR) y producción de etileno}

La clasificación de la guayaba como fruto climatérico o no climatérico es contradictoria: algunos autores consideran que la guayaba es no climatérica (Azzolini et al., 2005), mientras que otros la consideran climatérica (Kader, 2002; Bashir y Abu-Goukh, 2003; Singh y Pal, 2008a; Vieira et al., 2008; Solarte et al., 2010b; Yam Tzec et al.,
2010); esa condición puede ser un carácter varietal (Azzolini et al., 2005). Al respecto, Porat et al. (2009) estudiaron tres variedades de guayaba cultivadas en Israel, donde encontraron que la variedad tradicional Ben Dov exhibió un comportamiento clásico climatérico, mientras que la variedad King (pulpa rosada) presentó un comportamiento climatérico poco acentuado (suprimido) y la variedad Omri (pulpa blanca/amarillenta) fue aparentemente no climatérico ya que no mostró ningún aumento en la respiración y producción de etileno durante la maduración. Según Solarte et al. (2010b), este problema puede estar relacionado con el momento de la cosecha, ya que otras especies climatéricas como el melón, recolectados fuera de la madurez fisiológica, pueden comportarse como no climatéricos; además, también es probable el efecto varietal (genético) y de las condiciones ambientales. Según la tabla 1, las variedades climatéricas de la guayaba presentan valores de IR entre 31 y $1.400 \mathrm{mg}$ de $\mathrm{CO}_{2} \mathrm{~kg}^{-1} \mathrm{~h}^{-1}$, mientras Kader y Yahía (2011) la incluyen en el grupo de la producción de $\mathrm{CO}_{2}$ moderada, entre 70 y $150 \mathrm{mg}$ de $\mathrm{CO}_{2} \mathrm{~kg}^{-1} \mathrm{~h}^{-1}$, y la producción de etileno se encuentra entre $0,016 \mathrm{y}$ $94,0 \mu \mathrm{L} \mathrm{kg}^{-1} \mathrm{~h}^{-1}$, con pico climatérico respiratorio entre los días 3 y 12 después de la cosecha; el pico de producción de etileno se observa en los días cercanos al máximo climatérico respiratorio.

\section{Transpiración}

Según Kays y Paull (2004), la transpiración y el consumo de sustratos, son la razón de la pérdida de peso de los frutos durante la maduración en poscosecha. La pérdida de agua libre es una de las principales causas de deterioro, ya que se originan pérdidas cuantitativas directas, así como pérdidas cualitativas relacionadas con el aspecto, pérdida de brillo y de turgencia, cambios en la textura y consistencia de la fruta y en el valor nutricional (Kader, 2002, Yirat et al., 2009).

Suárez et al. (2009) en estudios de almacenamiento de guayaba tipo 'Criolla Roja' durante $20 \mathrm{~d}$ a tres temperaturas $\left(12,17\right.$ y $\left.27^{\circ} \mathrm{C}\right)$, encontraron 
Tabla 1. Intensidad respiratoria (IR) y producción etileno de frutos de guayaba en poscosecha.

\begin{tabular}{|c|c|c|c|c|c|}
\hline Variedad & $\begin{array}{l}\text { T. almacen } \\
\left({ }^{\circ} \mathrm{C}\right)\end{array}$ & $\begin{array}{c}\mathbb{R} \\
\left(\mathrm{mg} \text { de } \mathrm{CO}_{2} \mathrm{~kg}^{-1} \mathrm{~h}^{-1}\right)\end{array}$ & $\begin{array}{l}\text { Producción etileno } \\
\left(\mu \mathrm{L} \mathrm{C}_{2} \mathrm{H}_{4} \mathrm{~kg}^{-1} \mathrm{~h}^{-1}\right)\end{array}$ & Clasificación & Fuente \\
\hline \multirow{3}{*}{ Común } & 25 & & $0,016-0,25$ & & Singh y Pal (2008b) \\
\hline & & & $1,0-10,0$ & & Fischer et al. (2012) \\
\hline & 20 & & $1,0-20,0$ & & Kader (1999) \\
\hline Ben Dov & 20 & 65 a 92 (día 8) & 7,8 (día 7) & Climatérica & \multirow{3}{*}{ Porat et al. (2009) } \\
\hline King & 20 & 47 a 58 (día 9) & 4,1 (día 8) & $\begin{array}{l}\text { Climatérica } \\
\text { suprimida }\end{array}$ & \\
\hline Omri & 20 & 31 & $\leq 1,00$ & No climatérica & \\
\hline Paluma & 23 & 88,2 (en día 6) & & Climatérica & Bron et al. (2005) \\
\hline \multirow{4}{*}{ Pedro Sato } & 23 & 138-159 (día 8-12) & 3,25-3,70 (día 8-9) & Climatérica & Azzolini et al. (2005) \\
\hline & 22 & 80 (día 3) & 0,06 (día 3) & Climatérica & Vieira et al. (2008) \\
\hline & 8 & 45 (día 4) & 0,11 (día 12) & Climatérica & Vieira et al. (2008) \\
\hline & & 148,9 & & Climatérica & Basseto et al. (2005) \\
\hline $\begin{array}{l}\text { Media China } \\
\text { Primav.-Verano } \\
\text { Otoño-Invierno }\end{array}$ & $\begin{array}{l}25 \\
25 \\
\end{array}$ & $\begin{array}{l}\text { 122-175 (día 4) } \\
\text { 80-120 (día 8) }\end{array}$ & $\begin{array}{l}\text { 13,9-33,9 (día 5) } \\
11,7-23,9 \text { (día 9) }\end{array}$ & Climatérica & $\begin{array}{l}\text { Mercado-Silva et al. } \\
\text { (1998) }\end{array}$ \\
\hline $\begin{array}{l}\text { Regional Roja, Regional } \\
\text { Blanca, Ráquira Blanca } \\
\text { y Guavatá Victoria }\end{array}$ & 20 & $\begin{array}{l}500-1400 \\
\text { (dia 2-4) }\end{array}$ & 7,9-94,0 (día 4-8) & Climatéricas & Solarte et al. (2010b \\
\hline Allahabadi Safeda & 25 & 47,6 (en día 9) & 21,6 (en día 9) & Climatérica & Siddiqui et al. (1991) \\
\hline
\end{tabular}

que la pérdida de masa se incrementa a medida que aumenta la temperatura (para frutos con igual peso inicial, varía entre 32,9 y 89,9 g para la temperatura menor y mayor respectivamente), siendo además inversamente proporcional al estado de madurez del fruto. De acuerdo con Oliveira y Cereda (2003), los valores aceptables de pérdida de masa para guayabas recolectadas en estado de madurez fisiológica, varían entre 10 y $15 \%$.

Yam Tzec et al. (2009) realizaron pruebas a frutos de guayaba de las variedades Calvillo y Criolla sometidas a compresión, simulando el almacenamiento para dos posiciones del pedúnculo (horizontal y vertical) y almacenándolas posteriormente durante $4 \mathrm{~d}$ para determinar la pérdida de peso. Encontraron que al final del almacenamiento, la pérdida de peso fue mayor para los frutos sometidos a compresión en posición vertical (aproximadamente 10\% más) en comparación con los frutos ubicados en posición horizontal, lo cual indica que la posición del pedúnculo durante el almacenamiento, tiene influencia en la pérdida de masa y por lo tanto en la conservación de la calidad poscosecha.

\section{Cambios físicos}

\section{Firmeza del fruto}

La maduración poscosecha de algunas especies de frutos se caracteriza por el ablandamiento de la epidermis y la pulpa, causado por diferentes factores, entre ellos, la acción de las enzimas hidrolasas en la pared celular, las cuales actúan sobre la pectina; las celulasas, con actividad baja 
en frutos verdes, se incrementan rápidamente durante la maduración (Kays y Paull, 2004). Según Solarte et al. (2010b), las pectinasas (p.e. pectinmetil esterasa) remueven los grupos metilo del ácido galacturónico, permitiendo que las enzimas poligalacturonasas despolimericen la cadena de ácido poligalacturónico y reduzcan su peso molecular.
Diferentes autores indican que la firmeza de la guayaba, decrece con el avance del periodo poscosecha (Vazquez-Ochoa y Colinas-Leon, 1990; Bashir y Abu-Goukh, 2003; Jacomino et al., 2003; Azzolini et al., 2005; Vieira et al., 2008; Porat et al., 2009; Solarte et al., 2010b). La variación de la firmeza para diferentes variedades de guayaba se presenta en la tabla 2.

Tabla 2. Pérdida de peso y variación de la firmeza de frutos de guayaba en poscosecha.

\begin{tabular}{|c|c|c|c|c|}
\hline Variedad & T. almacén $\left({ }^{\circ} \mathrm{C}\right)$ & Firmeza & Pérdida peso & Fuente \\
\hline Común & 3,$5 ; 7$ y 11 & 25 a $7 \mathrm{~N}$ & 17,3 a 23,3 & Vazquez-Ochoa y Colinas-Leon (1990) \\
\hline Pulpa blanca y rosada & 22 & 2,13 a $0,20 \mathrm{~kg} \mathrm{~cm}^{-2}$ & & Bashir y Abu-Goukh (2003) \\
\hline Ben Dov & 20 & 21 a $8-10 N$ & & \multirow{3}{*}{ Porat et al. (2009) } \\
\hline King & 20 & 42 a $8-10 N$ & & \\
\hline Omri & 20 & 35 a $8-10 N$ & & \\
\hline Paluma & 23 & 132,5 a $20,06 \mathrm{~N}$ & & Cavalini et al. (2006) \\
\hline Kumagai & & 106,05 a $65,41 \mathrm{~N}$ & & Cavalini et al. (2006) \\
\hline Maamoura & 8 & & 9,4 en $15 d$ & Ismail et al. (2010) \\
\hline \multirow{5}{*}{ Pedro Sato } & 23 & 70 a $10 N$ & 3,5 en $8 d$ & Azzolini et al. (2005) \\
\hline & 22 & 50 a 5,24 N (4 d) & & Vieira et al. (2008) \\
\hline & 8 & 50 a $1,75 \mathrm{~N}(16 \mathrm{~d})$ & & Vieira et al. (2008) \\
\hline & 25 & 132,5 a $16,2 \mathrm{~N}$ & & Basseto et al. (2005) \\
\hline & 25 & 35,58 a $15,19 \mathrm{~N}$ & 6,6 en $6 \mathrm{~d}$ & Jacomino et al. (2003) \\
\hline $\begin{array}{l}\text { Media China } \\
\text { Primav.-Verano } \\
\text { Otoño-Invierno }\end{array}$ & $\begin{array}{l}25 \\
25 \\
\end{array}$ & $\begin{array}{l}35,9 \text { a } 4,7 \mathrm{~N} \\
39,1 \text { a } 4,9 \mathrm{~N} \\
\end{array}$ & & Mercado-Silva et al. (1998) \\
\hline Regional Roja & 20 & 4,42 a $0,58 \mathrm{~kg} \mathrm{~cm}^{-2}$ & 4,2 a $11,2(8-10$ d) & \multirow{4}{*}{ Solarte et al. (2010b } \\
\hline Regional Blanca & 20 & 3,60 a $0,47 \mathrm{~kg} \mathrm{~cm}^{-2}$ & 4,2 a $11,2(8-10 d)$ & \\
\hline Ráquira Blanca & 20 & 3,50 a $0,14 \mathrm{~kg} \mathrm{~cm}^{-2}$ & 4,2 a $11,2(8-10 d)$ & \\
\hline Guavatá Victoria & 20 & 2,79 a $0,62 \mathrm{~kg} \mathrm{~cm}^{-2}$ & 4,2 a $11,2(8-10$ d) & \\
\hline Allahabadi Safeda & 25 & & 11,35 a $12,63(15 d)$ & Siddiqui et al. (1991) \\
\hline
\end{tabular}




\section{Cambio de color}

Según Kader (2002), el cambio de color se presenta por degradación de la clorofila (debido a procesos oxidativos) y por la síntesis de otros pigmentos como antocianinas y carotenoides. En guayaba, el ángulo hue expresa significativamente el cambio en el color de la piel y es un buen indicador del estado de madurez poscosecha; las guayabas verdes muestran mayor ángulo hue que el fruto maduro (Mercado-Silva et al., 1998; Azzolini et al., 2004; Solarte et al., 2010b). El color expresado como ángulo hue $\left({ }^{\circ} \mathrm{h}\right)$ representa la tonalidad, que varía de $0^{\circ}$ en color rojo puro a $180^{\circ}$ en color verde puro (Solarte et al., 2010b). En guayaba tipo 'Criolla Roja' en estado de madurez fisiológica, Suárez et al. (2009) encontraron que la temperatura de refrigeración retrasa el proceso de maduración y por lo tanto, el cambio de color.

\section{Cambios bioquímicos}

\section{Carbohidratos y sólidos solubles totales (SST)}

Los carbohidratos forman parte de la estructura de las células y funcionan como reservas de energía; algunos carbohidratos simples como sacarosa, glucosa y fructosa, se consideran atributos de calidad de los frutos (Solarte et al., 2010b). Varios autores indican que los SST aumentan con el avance del periodo poscosecha, presentando valores que oscilan entre 5,0 y $13,2^{\circ}$ Brix (tabla 3 ).

Tabla 3. Variación en contenido de SST, ATT y ácido ascórbico de frutos de guayaba en poscosecha.

\begin{tabular}{|c|c|c|c|c|c|}
\hline Variedad & $\begin{array}{l}\text { T. almacén } \\
\left({ }^{\circ} \mathrm{C}\right)\end{array}$ & SST ( ${ }^{\circ}$ Brix) & ATT $(\%)$ & $\begin{array}{l}\text { Ácido ascórbico } \\
\text { (mg/100 g) }\end{array}$ & Fuente \\
\hline \multirow{4}{*}{ Común } & & & & $55-397$ & $\begin{array}{l}\text { Restrepo-Sánchez } \\
\text { et al. (2009) }\end{array}$ \\
\hline & & & & Hasta 2.000 & $\begin{array}{l}\text { Campbell (1984); Menzel } \\
\text { (1985); Martin et al. (1987) }\end{array}$ \\
\hline & 20 & & & $200-400$ & Kader (1999) \\
\hline & 3,$5 ; 7$ y 11 & 11,9 a $13,2(+)$ & 0,7 a $0,5(-)$ & 163 a $82(-)$ & $\begin{array}{l}\text { Vazquez-Ochoa } \\
\text { y Colinas-Leon (1990) }\end{array}$ \\
\hline $\begin{array}{l}\text { Cultivadas en } \\
\text { Georgia (USA) }\end{array}$ & & & & 350 & Yadava (1994) \\
\hline $\begin{array}{l}6 \text { selecciones de } \\
\text { pulpa rosa (México) }\end{array}$ & & 10,1 a $13,1(+)$ & & $83,3-185,8$ & $\begin{array}{l}\text { Mondragón Jacobo } \\
\text { et al. (2009) }\end{array}$ \\
\hline $\begin{array}{l}\text { De pulpa blanca y } \\
\text { rosada }\end{array}$ & 22 & 5,0 a $12,5(+)$ & $\begin{array}{l}(+) \text { climaterio } \\
\text { (día 8) y luego (-) }\end{array}$ & $\begin{array}{l}(-) \text { con el avance de la } \\
\text { maduración }\end{array}$ & $\begin{array}{l}\text { Bashir y Abu-Goukh. } \\
(2003)\end{array}$ \\
\hline Banahas & & 10,2 & \multirow{5}{*}{$\begin{array}{l}0,4 \text { a } 0,71 \\
\text { (valores puntuales) }\end{array}$} & \multirow{6}{*}{$\begin{array}{l}\text { Variaron entre } 52,80 \\
\text { y } 209,88 \text {. Mayores } \\
\text { contenidos en genotipos } \\
\text { de pulpa blanca } \\
\text { (valores puntuales) }\end{array}$} & \multirow{6}{*}{ Lima et al. (2002) } \\
\hline Lucknow 49 & & 10,9 & & & \\
\hline Patillo 2.1 & & 7,8 & & & \\
\hline Alabama Safed & & 7,8 & & & \\
\hline W.S. Florida & & 8,7 & & & \\
\hline Paluma & & 10,4 & 0,63 & & \\
\hline Paluma & & 7,5 a $8,5(+)$ & 0,78 a $0,47(-)$ & 62,80 a $84,94(+)$ & Cavalini et al. (2006) \\
\hline
\end{tabular}


Viene de la página anterior $\rightarrow$

\begin{tabular}{|c|c|c|c|c|c|}
\hline Variedad & $\begin{array}{c}\text { T. almacén } \\
\left({ }^{\circ} \mathrm{C}\right)\end{array}$ & SST ('Brix) & ATT $(\%)$ & $\begin{array}{c}\text { Ácido ascórbico } \\
\text { (mg/100 g) }\end{array}$ & Fuente \\
\hline Kumagai & & 6,0 a $6,7(+)$ & $0,59-0,54$ ( $\approx$ cte) & 103,28 a $128,36(+)$ & Cavalini et al. (2006) \\
\hline Guayaba dulce & 25 & 6,9 a $7,3(+)$ & & & Azzolini et al. (2004a) \\
\hline $\begin{array}{l}\text { Paluma, Rica, } \\
\text { Pedro Sato }\end{array}$ & 25,9 & $\approx 11$ & 0,59 & & Batista et al. (2012b) \\
\hline Maamoura & $8(15 d)$ & $\begin{array}{l}7,4 \text { a } 9,5(+) \\
\text { tratadas } \mathrm{CaCl}_{2}\end{array}$ & $\begin{array}{l}0,17 \text { a 0,25 (día 9) } \\
\text { a 0,2 (día 15) (+, -) }\end{array}$ & $\begin{array}{l}60,7 \text { a } 49,5(-) \\
\text { En todo tratamiento }\end{array}$ & Ismail et al. (2010) \\
\hline \multirow[b]{2}{*}{ Guayaba roja } & $37(15 d)$ & & 0,32 a $0,27(-)$ & & \multirow{2}{*}{ García et al. (2010) } \\
\hline & 9 & & 0,32 a $0,15(-)$ & & \\
\hline \multirow{3}{*}{ Pedro Sato } & 22 & 12,5 & 0,45 (día 12) (-) & & $\begin{array}{l}\text { Ferreira Soares et al. } \\
(2011)\end{array}$ \\
\hline & & 6,3 a $9,7(+)$ & 0,9 a $0,2(-)$ & & Hojo et al. (2007) \\
\hline & $25(6 d)$ & 8,82 a $9,52(+)$ & 0,60 a $0,54(-)$ & 60 a $52(-)$ & Jacomino et al. (2003) \\
\hline $\begin{array}{l}\text { Media China } \\
\text { Primav.-Verano } \\
\text { Otoño-Invierno }\end{array}$ & $25(7 d)$ & $\begin{array}{l}7,8 \text { a } 10,2(+) \\
11,1 \text { a } 12,1(+)\end{array}$ & $\begin{array}{l}0,73 \text { a } 0,54(-) \\
1,21 \text { a } 0,96(-)\end{array}$ & $\begin{array}{l}271 \text { a } 250(-) \\
341 \text { a } 284(-)\end{array}$ & Mercado-Silva et al. (1998) \\
\hline $\begin{array}{l}\text { Regional Blanca, } \\
\text { Regional Roja, Pera } \\
\text { y Manzana }\end{array}$ & $18(2-3 d)$ & $5,7-9.9$ & & $62,6-342,1$ & Rojas y Narváez (2009) \\
\hline $\begin{array}{l}\text { Regional Blanca, } \\
\text { Regional Roja, } \\
\text { Ráquira Blanca, } \\
\text { Guavatá Victoria }\end{array}$ & 20 & 8,6 a $11,97(+)$ & 0,80 a $0,56(-)$ & $\begin{array}{l}14,66-456,11 \\
\text { Sin tendencia clara }\end{array}$ & Solarte et al. (2010b) \\
\hline Guayaba Roja & & 7,9 a $10,5(+)$ & 0,82 a $0,74(-)$ & & \multirow{3}{*}{ González Cárdenas (2010) } \\
\hline Guayaba Blanca & & 8,9 a $9,8(+)$ & 0,71 a $0,60(-)$ & & \\
\hline Palmira ICA-1 & & 8,5 a $9,9(+)$ & 0,90 a $0,70(-)$ & & \\
\hline Allahabadi Safeda & $25(15 d)$ & 10 a $12(+)$ & & $\begin{array}{l}330 \text { a } 363 \text { (día 9) a } 95 \\
\text { (día 15) (+, -) }\end{array}$ & Siddiqui et al. (1991) \\
\hline
\end{tabular}

(+): Indica aumento (-): Indica disminución

Al respecto, Solarte et al. (2010b) encontraron que en las variedades colombianas Regional Blanca, Regional Roja, Guavatá y Ráquira, los SST aumentan (valores entre 8,6 y 12,0 ${ }^{\circ}$ Brix), así como el índice de madurez, indicando un incremento de azúcares junto con una disminución de ácidos durante el proceso de maduración en poscosecha, resultados que concuerdan con lo encontrado por González-Cárdenas (2010) para las variedades Guayaba Blanca, Guayaba Roja y Palmira ICA-1.

\section{Ácidos orgánicos y acidez total titulable (ATT)}

Los ácidos orgánicos desempeñan un papel crítico en aroma y sabor característico de los frutos, en el valor nutricional de los alimentos $y$ en el metabolismo poscosecha; algunos son componentes esenciales en el ciclo de los ácidos tricarboxílicos de la respiración y son una fuente de energía disponible que puede ser utilizada cuando otras fuentes se agotan (Solarte et al., 2010b). 
Para las diferentes variedades de guayaba, algunos autores indican que la ATT aumenta hasta el climaterio y luego disminuye (Bashir y AbuGoukh, 2003; Ismail et al., 2010), mientras que otros reportan que la ATT disminuye durante la poscosecha (Cavalini et al., 2006; Hojo et al., 2007; García et al., 2010; Ferreira Soares et al., 2011) o permanece constante (Cavalini et al., 2006) cuando el fruto se almacena a baja temperatura, con valores de ATT que oscilan entre 0,15 y $1,21 \%$ (tabla 3). Mercado-Silva et al. (1998), indican que los frutos cosechados en el periodo otoño-invierno presentan mayores valores de ATT que los frutos cosechados en primavera-verano y que la acidez tiende a disminuir en todos los casos después de 7 días de almacenamiento.

\section{pH}

En guayaba, los valores de $\mathrm{pH}$ varían entre 2,89 y 6,20, dependiendo de la variedad (Pereira et al., 2000). Laguado et al. (1999) indican que en frutos de guayaba de tipos Criolla Roja y San Miguel la concentración de ácidos libres disminuye por dilución, lo que aumenta el $\mathrm{pH}$ a medida que avanza el periodo poscosecha. Solarte et al. (2010b), en frutos de guayaba 'Regional Roja', 'Regional Blanca', 'Ráquira Blanca' y 'Guavatá Victoria' cultivadas en tres localidades colombianas, encontraron que el $\mathrm{pH}$ varió ligeramente durante la poscosecha, con valores que fueron desde 3,90 en la cosecha hasta 4,16 en el estado maduro. Un comportamiento similar fue encontrado por otros autores (Lima et al., 2002; González, 2010; Batista et al., 2012b).

De igual manera, González (2010) reporta para guayaba roja valores de $\mathrm{pH}$ que varían de 3,99 a 4,16; para guayaba blanca valores de 3,98 a 4,15 y para guayaba Palmira ICA-1 valores de 3,63 a 3,90. Batista et al. (2012b) indican que el pH varió de 3,92 a 4,25 para las variedades de guayaba Paluma, Rica y Pedro Sato cultivadas en Brasil, y almacenadas a temperatura ambiente $\left(25,9^{\circ} \mathrm{C}\right.$ y $66 \%$ HR) hasta completar la maduración pos- cosecha. Lima et al. (2002) encontraron valores de $\mathrm{pH}$ entre 3,72 y 4,22 para los diez cultivares de guayaba producidos en la región de Submédio São Francisco en Brasil.

\section{Contenido de ácido ascórbico (CAA)}

Varias investigaciones indican que la piel de la guayaba tiene mayor CAA y fenoles que la pulpa y que este varía entre cultivares (Bashir y Abu-Goukh, 2003; Lim et al., 2007). En la tabla 3 se relaciona el CAA reportado por diferentes autores para el fruto fresco, el cual puede llegar hasta $2.000 \mathrm{mg} / 100 \mathrm{~g}$. Generalmente, los cultivares de pulpa blanca contienen mayor CAA, fenoles y azucares (sacarosa, fructosa y glucosa) que los de pulpa roja (Bashir y Abu-Goukh, 2003; González-Aguilar et al., 2004); por su parte los frutos con semillas tienen mayores contenidos de fenoles y CAA que los frutos sin semillas (Lim et al., 2007).

Jacomino et al. (2003) para guayabas 'Pedro Sato' e Ismail et al. (2010) para frutos de guayaba 'Maamoura' encontraron que el CAA disminuye a medida que avanza la maduración. Los valores para el CAA, tienden a ser mayores a temperaturas más bajas (Vázquez-Ochoa y ColinasLeón, 1990). No obstante, Solarte et al. (2010b) afirman que el CAA no presenta una tendencia clara respecto al estado de maduración.

\section{Compuestos volátiles}

Según la variedad y el estado de madurez de la guayaba, se encuentran frutos con diferentes aromas y sabores (Soares et al., 2007). Steinhaus et al. (2008) indican que estos frutos son ricos en compuestos aromáticos volátiles, habiéndose identificado más de 400 componentes que pueden tener influencia en las características de olor. Los principales componentes dependen de la variedad y origen; así, para la variedad Cortibel de Brasil son: aldehídos, ésteres, monoterpenos, sesquiterpenos; para la variedad indefinida de Costa Rica son: terpenos y derivados terpénicos; 
para la variedad indefinida de Venezuela son: ésteres, sesquiterpenos, hidrocarburos monoterpénicos y para la variedad Wild de Florida son los hidrocarburos terpénicos (Jiménez et al., 2010).

Para las variedades cultivadas en Colombia, ICA-1 (guayaba pera) son: acetatos decinamilo, Z-3-hexenilo y alcohol cinamilico; para la variedad Glum Sali (guayaba manzana) son: E-2-hexanal, hexanal y tetradecano (Quijano et al., 1999). Para la variedad Regional Roja son: Z-3-hexenal, 3-sulfanil-hexanol, acetato de 3-sulfanil hexilo, furaneol y metoxifuraneol; para la variedad regional Blanca son: alcohol cinamilico, acetato de cinamilo, Z-3-hexenal, trans-4,5-epoxi-(E)-2-decenal, butanoato de etilo y furaneol (Jiménez et al., 2010).

\section{Patología y desórdenes fisiológicos}

\section{Enfermedades}

Con el avance de la maduración, la guayaba 'Pedro Sato' se vuelve más susceptible al ataque de patógenos debido a la disminución de la resistencia de la epidermis y de la pulpa (Azzolini et al., 2005). Según Droby et al. (2011), las enfermedades que se presentan son: antracnosis (causada por Colletrotrichum gloeosporioides y especies asociadas), pudrición por mucor (causada por Mucor hiemalis), pudrición por aspergilus (causada por Aspergillus niger), pudrición por rizopus (causada por Rhizopus stolonifer) y pudrición por fomopsis (causada por Phomopsis destructum). En guayabas, el agua caliente, el $\mathrm{H}_{2} \mathrm{O}_{2}$, el $\mathrm{CaCl}_{2}$ y el vapor de la hierba de limón que se aplica como un solo tratamiento, reducen la incidencia de la pudrición durante $15 \mathrm{~d}$ (Ismail et al., 2010).

\section{Daño por frío}

Según Kader (2002), los síntomas de daño por frío en guayabas en estado verde-maduro o con madurez parcial de consumo incluyen incapacidad para madurar normalmente, pardeamiento de la pulpa y de la piel, aumento en la inciden- cia y gravedad de las pudriciones cuando se les transfiere a temperaturas más altas. El estado de madurez es un factor que influye en la susceptibilidad al daño por frío; las guayabas maduras son menos sensibles al daño por frío que las que se encuentran en estado verde-maduro (Kader, 2002). Los síntomas de daño por frío en guayabas 'Allahabad Safeda' (pardeamiento de la corteza, desarrollo de picaduras en la superficie de la fruto y desecación), aparecieron después de dos semanas almacenadas a $5^{\circ} \mathrm{C}$ (Tiwari et al., 2006).

\section{ALGUNOS TRATAMIENTOS PARA PROLONGAR VIDA ÚTIL}

El tratamiento más efectivo para prolongar la vida útil poscosecha de productos agrícolas es el almacenamiento a baja temperatura, por encima de las temperaturas críticas para daño por frío (ParraCoronado y Hernández-Hernández, 2008). Se han utilizado diferentes técnicas buscando conservar la calidad de los frutos durante un mayor tiempo en la poscosecha, tales como el almacenamiento en atmósfera controlada y en atmósferas modificadas (Kader, 2002; Pal et al., 2007; Singh y Pal, 2008a), uso de 1-MCP (Blankenship y Dole, 2003; Singh y Pal, 2008b; Ortiz-Hernandez et al., 2010), uso de emulsiones de recubrimiento y tratamiento hidrotérmico (Jacomino et al., 2003; Vieira et al., 2008; Espinoza-Zamora et al., 2010; Salinas-Hernández et al., 2010; Ferreira Soares et al., 2011), uso de antioxidantes (Jayachandran et al., 2007), irradiaciones gamma (Pimentel, 2007; De Campos et al., 2011), $\mathrm{CaCl}_{2}$, peróxido de hidrógeno $\left(\mathrm{H}_{2} \mathrm{O}_{2}\right)$, fumigación con limoncillo (Castellano et al., 2005a, 2005b; Ismail et al., 2010) y posición del pedúnculo durante el almacenamiento (Yam Tzec et al., 2009). Estos tratamientos poscosecha utilizados para guayabas retrasan el proceso de maduración y, por tanto, permiten conservar la calidad de los productos durante un mayor tiempo; sin embargo, se ha demostrado que son más efectivos cuando se combinan con almacenamiento a bajas temperaturas. 


\section{Almacenamiento refrigerado}

Según González-Aguilar et al. (2004) y Kader (2002), los frutos de guayaba son muy susceptibles a los daños por frío y a las enfermedades; no obstante, en plena madurez de consumo estos son menos sensibles al daño por frío que los que se encuentran en estado verde-maduro; la temperatura óptima para guayabas verde-maduras y parcialmente maduras (vida potencial de almacenamiento de dos o tres semanas) está entre 8 y $10^{\circ} \mathrm{C}$ y para guayabas completamente maduras (vida potencial de almacenamiento de una semana) está entre 5 y $8^{\circ} \mathrm{C}$; la tasa de respiración de la guayaba almacenada a $10^{\circ} \mathrm{C}$ está entre 4 y $30 \mathrm{~mL}$ $\mathrm{CO}_{2} \mathrm{~kg}^{-1} \mathrm{~h}^{-1}$ y a $20^{\circ} \mathrm{C}$ está entre 10 y $70 \mathrm{~mL} \mathrm{CO}_{2}$ $\mathrm{kg}^{-1} \mathrm{~h}^{-1}$ (Kader, 2002).

De igual manera, la temperatura de almacenamiento influye en la pérdida de masa de los frutos de guayabas, incrementándose a medida que aumenta la temperatura y disminuye la humedad relativa (HR) del sistema de almacenamiento (Vazquez-Ochoa y Colinas-Leon, 1990). La temperatura de almacenamiento afecta el comportamiento fisiológico y bioquímico de los frutos, de tal manera que a bajas temperaturas se retarda el proceso de maduración poscosecha, el cual se manifiesta por disminución de la intensidad respiratoria (tabla 1), retardo en la pérdida de firmeza del fruto (tabla 2) y en los cambios correspondientes a SST, ATT y pH. Algunos autores reportan que la ATT disminuye durante la poscosecha o permanece constante cuando el fruto se almacena a baja temperatura, con valores de ATT que oscilan entre 0,15 y $1,21 \%$ (tabla 3 ).

\section{CONCLUSIONES}

Los diferentes estudios poscosecha realizados indican que los frutos de guayaba son muy susceptibles al daño por frío, debiéndose almacenar a una temperatura adecuada de $5-10^{\circ} \mathrm{C}$. La clasificación de la guayaba como fruto climatérico o no climatérico es contradictorio; algunos autores indican que es climatérico, mientras otros afirman que es no-climatérico, según la variedad. A medida que transcurre el periodo poscosecha aumenta la pérdida de masa, los sólidos solubles y el $\mathrm{pH}$ y disminuye la firmeza del fruto y el ángulo hue. La tendencia de la ATT y del CAA no es clara, ya que algunos autores indican que estos aumentan hasta el climaterio y luego disminuye, mientras que otros reportan que disminuyen durante almacenamiento o permanecen constantes, comportamiento que probablemente esté influenciado por las características varietales, edad de la planta y por las condiciones climáticas y de cultivo a que hayan estado expuestos.

El comportamiento fisiológico y las características poscosecha de los frutos (SST, ATT, CAA, firmeza, entre otros), pueden variar en una misma localidad de acuerdo con el cultivar, la edad de la planta, la época del año, las condiciones de cultivo, de almacenamiento y manejo poscosecha de los frutos.

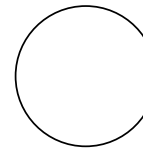

Agronet. Reportes estadísticos. En: http://www.agronet.gov.co; consulta: abril de 2014.

Azzolini, M., A.P. Jacomino y L.1. Urbano. 2004. Índices para avaliar qualidade póscolheita de goiabas em diferentes estádios de maturação. Pesq. Agropec. Bras. 39(2), 139-145.

Azzolini, M., A.P. Jacomino, I.U. Bron, R.A. Kluge y M. Schavinato. 2005. Ripening of 'Pedro Sato' guava:

\section{REFERENCIAS BIBLIOGRÁFICAS}

study on its climateric or nonclimateric nature. Braz. J. Plant Physiol. 17(3), 299-306. Doi: 10.1590/ S1677-04202005000300004

Bashir, H.A. y A.B. Abu-Goukh. 2003. Compositional changes during guava fruit ripening. Food Chem. 80, 557-563. Doi: 10.1016/S0308-8146(02)00345-X

Batista, P.F., M.A.C. de Lima, D.C.G. da Trindade, R.V. Façanha y R.E. Alves. 2012a. Bioactive compounds 
and antioxidant activity in guava fruit cultivated in Sub-Midlle São Francisco Valley, Brazil. En: Abstracts $3^{\text {rd }}$ International Symposium on Guava and Other Myrtaceae, Petrolina, PE, Brasil.

Batista, P.F., M.A.C. de Lima, D.C.G. da Trindade, A.L.S. Araújo y R.E. Alves. 2012b. Chemical characterization of guava fruit produced in submiddle of São Francisco Valley, Brazil. En: Abstracts $3^{\text {rd }}$ International Symposium on Guava and other Myrtaceae. Petrolina, PE, Brasil.

Blankenship, S.M. y J.M. Dole. 2003. 1-Methylcyclopropene: a review. Postharv. Biol. Technol. 28, 1-25. Doi: 10.1016/S0925-5214(02)00246-6

Campos, A.J., E. Fujita, L.C. Neves, R.L. Vieites y E.A. Chagas. 2011. Radiação gama e atmosfera modificada passiva na qualidade de goiabas "Pedro Sato". Rev. Bras. Frutic. 33(especial 1), 350-356.

Castellano, G., O. Quijada, R. Ramírez y E. Sayago. 2005a. Comportamiento poscosecha de frutas de guayaba ( $P$. guajava L.) tratados con cloruro de calcio y agua caliente a dos temperaturas de almacenamiento. Rev. Iberoamer. Tecnol. Postcosecha 6(2), 78-82.

Castellano, G., O. Quijada, R. Ramírez y E. Sayago. 2005b. Efecto de tratamientos poscosecha sobre la calidad de frutas de guayaba (P. guajava L.) Proc. Interamer. Soc. Trop. Hort. 48, 111-114.

Droby, S., M. Israel y N. Benkeblia. 2011. Postharvest pathology of tropical and subtropical fruit and strategies for decay control. pp. 194-223. En: Yahia, E.M. (ed.) Postharvest biology and technology of tropical and subtropical fruits. Vol. 3. Woodhead Publishing, Oxford, UK.

Espinal, M., J. Olaya, P. Restrepo, K. Silva y F. Parada. 2010. La guayaba, fuente de fenoles con actividad antioxidante. pp. 177-185. En: Morales, A.L. y L.M. Melgarejo (eds.). 2010. Desarrollo de productos funcionales promisorios a partir de la guayaba (P. guajava L.) para el fortalecimiento de la cadena productiva. Facultad de Ciencias, Universidad Nacional de Colombia, Bogotá.

Espinoza-Zamora, J., R. Baez-Sañudo, C. Saucedo-Veloz y E. Mercado-Silva. 2010. Effect of application of waxes with vegetable oil and sucrose on the quality of Mexican guava cv. 'Media China'. Acta Hort. 849, 393-400.

Ferreira Soares, N.F., D.F. Pereira Silva, G. Geany Peruch Camilloto, C.P. Oliveira, N.M. Pinheiro y E.A. Alves Medeiros 2011. Antimicrobial edible coating in post-harvest conservation of guava. Rev. Bras. Frutic. (Vol. Esp.), 281-289. Doi: 10.1590/S010029452011000500035
Fischer, G., L.M. Melgarejo y D. Miranda. 2012. Guayaba (P. guajava L.). pp. 526-549. En: Fischer, G. (ed.). 2012. Manual para el cultivo de frutales en el trópico. Produmedios, Bogotá.

Giovannoni, J. 2001. Molecular biology of fruit maturation and ripening. Annu. Rev. Plant Physiol. Plant Mol. Biol. 52, 725-749. Doi: 0.1146/annurev. arplant.52.1.725

González, I.A. 2010. Caracterización química del color de diferentes variedades de guayaba ( . guajava L.) colombiana. Tesis de maestría. Departamento de Química, Facultad de Ciencias, Universidad Nacional de Colombia, Bogotá.

González-Aguilar, G.A., M.E. Tiznado-Hernández, M. Zavaleta-Gatica y M.A. Martínez-Téllez. 2004. Methyl jasmonate treatments reduce chilling injury and activate the defense response of guava fruits. Biochem. Biophys. Res. Comm. 313, 694701. Doi: 10.1016/j.bbrc.2003.11.165

Ismail, O.M., A.A. Eman Abd El-Moniem, A.S.E. AbdAllah y M.A.A. El-Naggar. 2010. Influence of some post-harvest treatments on guava fruits. Agric. Biol. J. N. Am. 1(6), 1309-1318. Doi: 10.5251/abjna.2010.1.6.1309.131

Jacomino, A.P., R. Martínez Ojeda, R.A. Kluge y J.A. Scarpare Filho. 2003. Conservação de goiabas tratadas com emulsões de cera de carnauba. Rev. Bras. Frutic. 25(3), 401-405.

Jayachandran, K.S., D. Srihari y Y.N. Reddy. 2007. Postharvest application of selected antioxidants to improve the shelf life of guava fruit. Acta Hort. $735,627-632$.

Jiménez, A., H. Barbosa, A.L. Morales y C. Osorio. 2010. Deshidratación de la guayaba, una opción para su aprovechamiento integral. pp. 125-138. En: Morales, A.L. y L.M. Melgarejo (eds.). Desarrollo de productos funcionales promisorios a partir de la guayaba ( $P$. guajava L.) para el fortalecimiento de la cadena productiva. Facultad de Ciencias, Universidad Nacional de Colombia, Bogotá.

Kader, A.A. 2002. Postharvest biology and technology: An overview. pp. 39-48. En: Kader, A.A. (ed.). Postharvest technology of horticultural crops. 3a. ed. Publication 3311. Agriculture and Natural Resources, University of California, Oakland, CA.

Kader, A.A. y E.M. Yahia. 2011. Postharvest biology of tropical and subtropical fruits. pp. 79-111. En: Yahia, E.M. (ed.). Postharvest biology and technology of tropical and subtropical fruits. Vol. 1. Woodhead Publishing Limited, Cambridge, UK.

Kays, S.J. y R.E. Paull. 2004. Postharvest biology. Exxon Press, Athens, GA. 
Laguado, N., E. Pérez, C. Alvarado y M. Marín. 1999. Características fisicoquímicas y fisiológicas de frutos de guayaba de los tipos Criolla Roja y San Miguel procedentes de dos plantaciones comerciales. Rev. Fac. Agron. (LUZ) 16(4), 382-397.

Lim, Y.Y., T.T. Lim y J.J. Lee. 2007. Antioxidant properties of several tropical fruits: A comparative study. Food Chem. 103, 1003-1008. Doi: 10.1016/j.foodchem.2006.08.038

Lima, M. A. C., J.S. de Assis y L. Gonzaga Neto. 2002. Caracterização dos frutos de goiabeira e seleção de cultivares na Região do Submédio São Francisco. Rev. Bras. Frutic. 24(1), 273-276.

Mendes-Pereira, F. y R. Kavati. 2011. Contribuição da pesquisa científica brasileira no desenvolvimento de algumas frutíferas de clima subtropical. Rev. Bras. Frutic. (Vol. Esp.), 92-108.

Mercado-Silva, E., P.B. Bautista y M.A. Garcia-Velasco. 1998. Fruit development, harvest index and ripening changes of guavas produced in Central Mexico. Postharv. Biol. Technol. 13(2), 143-150. Doi: 10.1016/S0925-5214(98)00003-9

Oliveira, M.A. y M.P. Cereda. 2003. Pós-colheita de Pêssegos (Prunus persica L. Batsch) revestidos com filme a base de amido como alternativa à cera comercial. Ciência Tecnol. Alimentos 23, 20-33. Doi: 10.1590/S0101-20612003000400006

Ortiz-Hernandez, G.R., J. Espinoza-Hernández, C. Saucedo-Veloz y E. Mercado-Silva. 2010. Effect of 1-MCP on shelf-life and volatile production of Mexican guava fruits (cv. 'Media China'). Acta Hort. 849, 387-392.

Pal, R.K., S.P. Singh, C.P. Singh y R. Asre. 2007. Response of guava fruit (Psidium gajava L. Cv. Lucknow-49) to controlled atmosphere storage. Acta Hort. 735, 547-554.

Parra-Coronado, A. y J.E. Hernández-Hernández. 2008. Fisiología postcosecha de frutas y hortalizas. $4^{\mathrm{a}} \mathrm{ed}$. Facultad de Ingeniería, Universidad Nacional de Colombia, Bogotá.

Pereira W.E., F.A. D'Araujo, D. Lopes, B.C. Horts, P.R. Cecon y R. Santos. 2000. Rendimento e algumas características físicoquímicas dos frutos de seis variedades de goiabeira desenvueltos em condições de déficit hídrico. Rev. Ceres 47(272), 349-362.

Pereira, F.M., C.A. Carvalho y J.C. Nachtigal. 2003. Século XXI: nova cultivar de dupla finalidade. Rev. Bras. Frutic. 25(3), 498-500. Doi: 10.1590/S010029452003000300034

Pereira, F.M. y J.C. Nachtigal. 2009. Melhoramento genético da goiabeira. pp. 371-398. En: Natale, W.,
D.E. Rozane, H.A. de Souza y D.A. de Amorim (eds.). Cultura da goiaba: do plantio à comercialização. Vol. 2. Jaboticabal, Brasil.

Pérez-Gutierrez, R.M., S. Mitchel y R.V. Solis. 2008. Psidium guajava: A review of its traditional uses, phytochemistry and pharmacology. J. Ethnopharmacol. 117, 1-27. Doi: 10.1016/j.jep.2008.01.025

Pimentel, R.M.A. 2007. Qualidade pós-colheita da goiaba-vermelha (P. guajava L.) submetida ao tratamento quarentenário por irradiação gama. Tese (Doutorado em Ciências, Área de Concentração: Energia Nuclear na Agricultura) - Centro de Energia Nuclear na Agricultura da Universidade de São Paulo, Piracicaba, Brasil.

Porat, R., B. Weiss, I. Zipori y A. Dag. 2009. Postharvest longevity and Responsiveness of guava varieties with distinctive climacteric behaviors to 1-Methylcyclopropene. HortTechnol. 19(3), 580-585.

Quijano, C., M. Suárez y C. Duque. 1999. Constituyentes volátiles de dos variedades de guayaba ( $P$. gua java L.): Palmira ICA-1 y Glum Sali. Rev. Colomb. Quim. 28, 55-63.

Restrepo-Sánchez, D.C., C.E. Narváez-Cuenca y L.P. Restrepo-Sánchez. 2009. Extracción de compuestos con actividad antioxidante de frutos de guayaba cultivada en Vélez-Santander, Colombia. Quim. Nova 32(6), 1517-1522

Salinas-Hernández, R.M., F. Ulín-Montejo y C. SaucedoVeloz. 2010. Effect of waxing and temperature of storage on the conservation of guava ( $P$. guajava L.) cultivar 'Media China'. Acta Hort. 849, 401-408.

Singh, S.P. y R.K. Pal. 2008a. Controlled atmosphere storage of guava ( $P$. guajava L.) fruit. Postharv. Biol. Technol. 47(3), 296-306. Doi: 10.1016/j.postharvbio.2007.08.009

Singh, S.P. y R.K. Pal. 2008b. Response of climacteric type guava ( $P$. guajava $\mathrm{L})$ to postharvest treatment with 1-MCP. Postharv. Biol. Technol. 47, 307-314. Doi: 10.1016/j.postharvbio.2007.08.010

Singh, S.P. 2011. Guava (P. guajava L.). pp. 213-245. En: Yahia, E.M. (ed.). Postharvest biology and technology of tropical and subtropical fruits. Vol. 3. Woodhead Publishing Limited, Cambridge, UK.

Soares, F.D., T. Pereira, M.O. Maio Marques y A.R. Monteiro. 2007. Volatile and non-volatile chemical composition of the white guava fruit (Psidium guajava) at different stages of maturity. Food Chem. 100, 15-21. Doi: 10.1016/j.foodchem.2005.07.061

Solarte, M.E., H.M. Romero y L.M. Melgarejo. 2010a. Caracterización ecofisiológica de la guayaba de la hoya del rio Suárez. pp. 25-56. En: Morales, A.L. y 
L.M. Melgarejo (eds.). 2010. Desarrollo de productos funcionales promisorios a partir de la guayaba (P. guajava L.) para el fortalecimiento de la cadena productiva. Facultad de Ciencias, Universidad Nacional de Colombia, Bogotá.

Solarte, M.E., M.S. Hernández, A.L. Morales, J.P. Fernández y L.M. Melgarejo. 2010b. Caracterización fisiológica y bioquímica del fruto de guayaba durante la maduración. pp. 85-119. En: Morales, A.L. y L.M. Melgarejo (eds.). 2010. Desarrollo de productos funcionales promisorios a partir de la guayaba ( $P$. guajava L.) para el fortalecimiento de la cadena productiva. Facultad de Ciencias, Universidad Nacional de Colombia, Bogotá.

Solarte, M.E., O. Insuasty y L.M. Melgarejo. 2010c. Calendario fenológico de la guayaba en la hoya del río Suárez. pp. 59-82. En: Morales, A.L. y L.M. Melgarejo (eds.). Desarrollo de productos funcionales promisorios a partir de la guayaba (Psidium guajava L.) para el fortalecimiento de la cadena productiva. Facultad de Ciencias, Universidad Nacional de Colombia. Bogotá.

Steinhaus, M., D. Sinuco, J. Polster, C. Osorio y P. Schieberle. 2008. Characterization of the aromaactive compounds in pink guava ( $P$. guajava L.) by application of the aroma extract dilution analysis. J. Agr. Food Chem. 56, 4120-4127. Doi: 10.1021/ jf800524

Suárez, J., M. Pérez de Camacaro y A. Giménez. 2009. Efecto de la temperatura y estado de madurez sobre la calidad poscosecha de la fruta de guayaba (Psidium guajava L.) procedente de MERCABAR, estado Lara, Venezuela. Rev. Cient. UDO Agr. 9(1), 60-69.

Tiwari, S., D.K. Tandon y E.B. Esguerra. 2006. Chilling injury as an indicator of critical temperature for cold storage of guava (P. guajava L.) cv. Allahabad Safeda. Int. J. Postharv. Technol. Innov. 1(2), 170-177. Doi: 10.1504/IJPTI.2006.011659
Vazquez-Ochoa, R.I. y M.T. Colinas-Leon. 1990. Changes in guavas of three maturity stages in response to temperature and relative humidity. HortScience 25(1), 86-87.

Vieira, S.M.J., S.M. Couto, P.C. Corrêa, A.E.O. dos Santos, P.R. Cecom y D.J.P. da Silva. 2008. Características físicas de goiabas ( $P$. guajava L.) submetidas a tratamento hidrotérmico. R. Bras. Eng. Agríc. Ambiental 12(4), 408-414. Doi: 10.1590/S141543662008000400012

Wilson, P.G., M.M. O'Brien, P.A. Gadek y C.J. Quinn. 2001. Myrtaceae revisited: a reassessment of infrafamilial groups. Amer. J. Bot. 88(11), 2013-2025. Doi: $10.2307 / 3558428$

Wills, R., B. McGlasson, D. Graham y D. Joyuce. 2007. Postharvest - An introduction to the physiology and handling of fruit, vegetables and ornamentals. $5^{\mathrm{a}}$ ed. Cabi International, Wallingford, UK.

Yadava, U.L. 1996. Guava (P. guajava L.): An exotic tree fruit with potential in the southeastern United States. HortScience 31(5), 789-794.

Yam Tzec, J.A., C.A. Villaseñor Perea, E. Romantchik Kriuchkova, M. Soto Escobar y M.A. Peña Peralta. 2009. Análisis de frutos de guayaba (psidium guajava L.) bajo compresión y su relación con los procesos fisiológicos. Ingeniería Agrícola y Biosistemas $1(1), 63-70$

Yam Tzec, J.A., C.A. Villaseñor Perea, E. Romantchik Kriuchkova, M. Soto Escobar y M.A. Peña Peralta. 2010. Una revisión sobre la importancia del fruto de guayaba ( $P$. guajava L.) y sus principales características en la postcosecha. Rev. Cienc. Técn. Agropec. 19(4), 74-82.

Yirat, M., A. García, A. Hernández, A. Calderín y N. Camacho. 2009. Evaluación de la calidad de la guayaba, variedad Enana Roja EEA-1-23, durante el almacenamiento a temperatura ambiente. Rev. Cienc. Técn. Agropec. 18(2), 70-73. 